\title{
Research on BIM Technology Modeling Method in Construction of Bridge Engineering
}

\author{
Ma Shaoxiong ${ }^{1,2}$, a, Chen Cunli ${ }^{1}$ and Zhao Qin ${ }^{1}$ \\ ${ }^{1}$ Faculty of Civil Engineering and Architecture, Xi' an University of Technology, Xi' an 710048, China \\ 2Shan Xi Railway Institute, Weinan, 714000, China \\ a 2258824468@qq.com
}

\begin{abstract}
Keywords: Bridge construction; BIM; CATIA; Three-dimensional modeling.
Abstract. BIM technology covers a large number of information related to project which is serviced in the design of the construction project, construction installation, operation and the whole lifecycle by establishing the digital design of BIM model and plays a huge role in improving production efficiency, ensuring the quality of production, saving cost, shortening the construction period and other advantages. On the basis of a bridge engineering background, this paper introduces the characteristics and advantages of CATIA software, discusses the basic idea of the three-dimensional model of bridge engineering construction stage, analyzes the key and difficult problems in the process of modeling and puts forward the solution. Through the BIM three-dimensional modeling, the construction side of the project grasps more clearly particularly in the details of the situation. And the establishment of BIM model will directly meet the needs of construction management.
\end{abstract}

\section{Introduction}

Building information modeling (BIM) is used as an innovative tool and production methods which has triggered a huge change in Europe and the United States and other developed countries ,is aimed as including construction projects in the relevant information and data based on the three-dimensional Model, is the whole life cycle of the building structure to carry out a full range of management and collaborative work, is the construction of the physical characteristics and functional characteristics of the digital bearing and visual expression. ${ }^{[1]} \mathrm{BIM}$ technology injects fresh vitality to the entire infrastructure industry in the development of municipal industry. As an important component of the national infrastructure and the node of the traffic system, the bridge engineering is characterized by its complicated design, frequent change, long construction period and difficulty. With the rapid development of the economic, large and super large bridge projects increase, people increasingly high demand on the project's economy, safety, construction efficiency, as well as the quality of the bridge, life expectancy, landscape requirements. Relying on the traditional two-dimensional design methods and traditional construction methods cannot meet people's current high standards of the bridge requirements and expectations, the application of information technology is increasingly urgent and necessary. ${ }^{[2]}$

In the past, exploring the application of BIM technology in bridge engineering project mainly discusses how to combine BIM technology with each stage of bridge project at theoretical level, and how to use BIM software to carry out practical modeling research in engineering construction stage is less. Based on a bridge project as the engineering background, this paper discusses the application of CATIA software in the construction phase of the bridge to carry out the three-dimensional modeling of the specific process. Reasonable and effective three-dimensional model is the application of BIM technology in the construction process of all aspects of scientific guidance, which ensures the construction quality and construction efficiency, and makes the project quality and safety, the premise of the maximum economic benefits.

\section{Introduction of CATIA Software}

CATIA software is the pioneer of Dassault Systèmes, leading the world in product design and innovation solutions. CATIA software has a unified user interface, data management and compatible 
with the database and application program interface. Feature modeling and parametric modeling techniques are used to allow variable design to be automatically assigned or specified by the user through parametric design, geometry or functional constraints. It is favored by many users due to it has excellent knowledge of intelligence, mechanical product design, finite element analysis, NC programming, digital virtual prototyping and other powerful functional modules which is widely used in aviation, automotive and other fields.

At present, most CAD / CAM software has the function of parametric modeling, but some need to be realized by interface programming with software, which requires the developer to have a high level of programming; some of them cannot be operated by visualization, which requires the user have a good command of the software and operation.V5 CATIA makes users complete the three-dimensional parametric modeling efficiently through the visual characteristics of trees and a variety of visual tools. ${ }^{[3-4]}$

In this paper, based on the modeling practice of a bridge project construction phase, this paper focuses on the three-dimensional modeling method of CATIA software which is suitable for bridge engineering.

\section{The modeling scheme of the project}

The reasonable three-dimensional model of bridge engineering is the basis and prerequisite of applying BIM technology to guide the construction of digital information, construction plan and schedule optimization and the dynamic management of construction process resources in bridge construction. According to the characteristics of this project, the design modeling process is as follows.

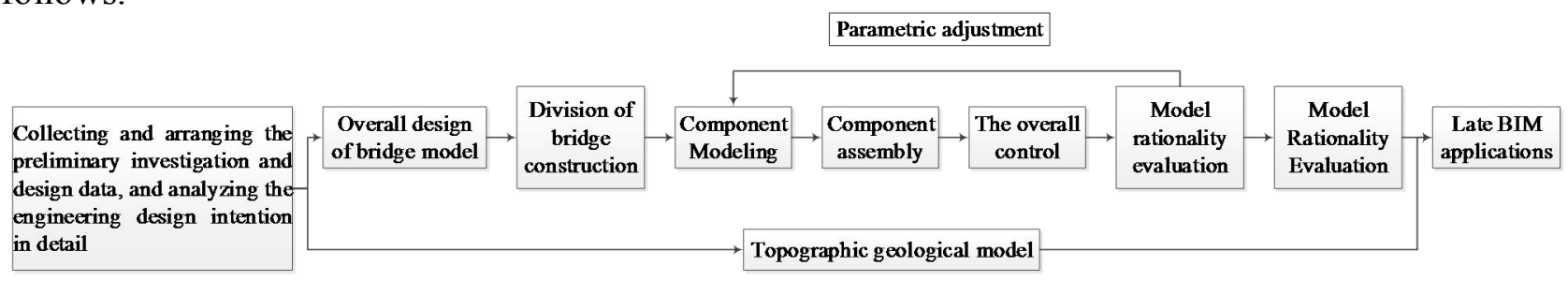

Fig.1.Application of CATIA Software to the Overall Project of Bridge Engineering Modeling

\section{Detailed modeling process}

\section{Terrain geological modeling}

Terrain geology model is based on terrain cloud point, and the formation of terrain through the three-dimensional network structure, after the terrain through the formation of three-dimensional terrain entity entities, the switch between different angles can clearly show the ground fluctuations ${ }^{[5]}$. The difficulty of this step is how to achieve AutoCAD information data and CATIA data exchange. To solve this problem, the project has developed a CATIA software data interface, and developed a data conversion plug-in to achieve the AutoCAD and CATIA data exchange. The three-dimensional geological model is suitable for the later BIM application which can meet the need of obtaining the geological excavation surface, cutting the geological excavation body, calculating the excavation and excavation side, and cutting the vertical and horizontal sections of the geological section. 


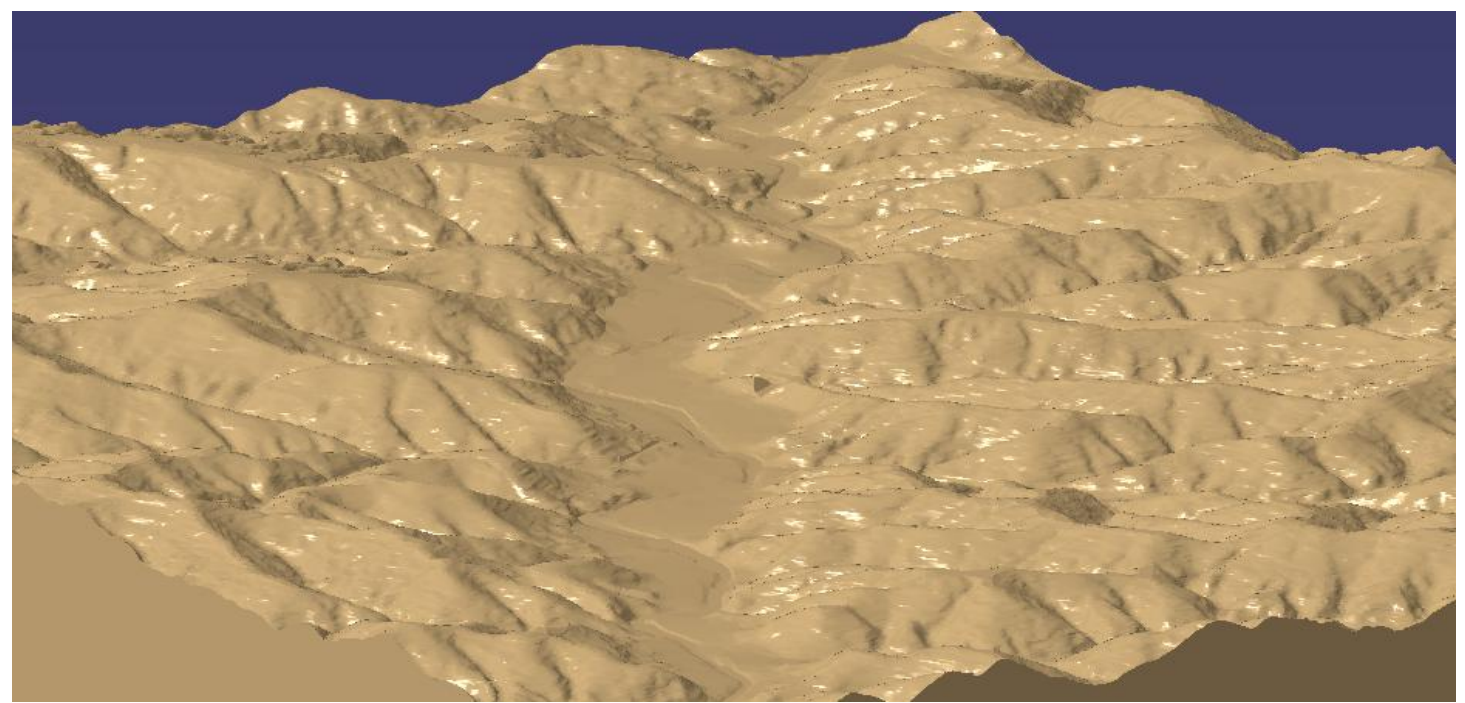

Fig.2. Geological body model

\section{Overall design of bridge model}

CATIA V5 not only has system-defined parameters, but also user-defined parameters. Designers can easily customize the various parameters and formulas that constrain these parameters by means of user-defined parameters and formulas. Among them, the user defined formula is the link between the system parameters and the user parameters in V5 CATIA. After the definition of user parameters, the designer can set up the corresponding formula for the corresponding characteristic parameters in the 3D model, so as to drive the system parameters through the user parameters, and then control the size of the graph ${ }^{[4]}$. It is easy to solve the bridge design problem of skewed $30^{\circ} \mathrm{T}$ beam in this project by using the CAD software. In order to determine the model modeling base and ensure the model information and the construction site data dock accurately by using the real bridge construction coordinates. Using CAD software powerful three-dimensional surface modeling function, to achieve the edge beam beam width of the outer edge of the curve with the line changes. According to the above design principles to model, on the one hand can improve the accuracy of the model, on the other hand, the user can query the model coordinates directly to guide the construction.

\section{Component design}

The main structure of the bridge engineering main components generally include the main beam, support, foundation, connection member, connection node, bridge deck, auxiliary equipment, etc. Therefore, before the BIM modeling of the main structure of the bridge, combined with the bridge construction method, the main components of the bridge division, and according to the design drawings for component modeling. In view of the existing assembly function of CATIA software, it is very inconvenient to locate the relative position relation between the components of the bridge. In this project, firstly, the control points of the bridge line control information space in the design scheme are determined. Reference to this information modeling, and finally combined directly into the entire structure. After deeply familiar with the design drawings, the paper focuses on the modeling of the $\mathrm{T}$ beams with a simple supported skew beam.

\section{Efficient and fast modeling of the super copy}

In bridge engineering, most of the components are structural members, and most of them are not standard components. However, in a specific bridge project, there are usually a large number of difference and have a certain variation of the sub-elements. ${ }^{[3]}$ Therefore, the project in the modeling, analysis and induction of similar structural features of the components, and these components embedded in the parts ahead of time, and then through the extraction of these knowledge, and in-house configuration, use the same type of component creation Template super copy and select the 
instantiation command to create other components of the same type, in order to achieve knowledge reuse, to ensure the accuracy of modeling and improve modeling efficiency.

\section{Conclusion}

BIM model, as the main carrier of bridge engineering data, has become the basis of BIM technology to guide all aspects of bridge engineering construction. The quality of the model is directly related to the success or failure of BIM in the construction phase. Based on a bridge engineering modeling practice, this paper focuses on the general idea of building a 3D parametric model based on CATIA software and using visualization tools in its knowledge engineering module. Among them, how to partition and establish the component of BIM determines the convenience and accuracy of the extraction of the relevant data of the project. The application of super copy achieves the reuse of knowledge and improve the efficiency of modeling greatly. A consistent with the construction process of the model, the progress of the construction phase of the follow-up, quality and safety issues will be more accurate tracking. A model in line with the construction process, the construction phase of the progress of follow-up, quality and safety issues will be more accurate tracking.

\section{Acknowledgements}

The authors wish to acknowledge the support of the National Natural Science Foundation of China (No.51408488), Shaanxi Science and technology innovation project of Province(2015KTZDGY01-04), Science and technology research and development program of Shaanxi Province(2012K12-03-05).

\section{References}

[1]. Gao Jing-jing, Zou Jun-zhen, Zhang Jin-yue. Application of BIM Technology in Bridge Construction[J]. Western China Communications Science \& Technology, 2016(1).

[2]. Wang Bin. Research On The Application Of Building Information Modeling In Bridge Engineering[D]. Nanjing, Southeast University, 2015.

[3]. Huang Jun-xuan, Zhang Lei, Ye Yi. 3D Modeling Method of Large Bridge based on CATIA [J].Journal of Information Technology in Civil Engineering and Architecture, 2012(4):51-55.

[4]. Chen Jing-xin,Xu Jing,Lu Guo-min, et al. Three dimensional parametric modeling method based on CATIA and its application[J]. Journal of Machine Design, 2003, 20(8):48-50.

[5]. Du Xiao, Ren You-feng, Shang An'fan. Application of 3D CATIA in Design of Cable-stayed Bridge with Unequal Span[J].Northwest Hydropower, 2016(3). 